\title{
Invasive Trichosporonosis in a Critically Ill ICU Patient: Case Report
}

\author{
Maja Tomić Paradžik ${ }^{1^{*}}$, Josip Mihić ${ }^{2}$, Jasminka Kopić ${ }^{3}$ and Emilija Mlinarić Missoni ${ }^{4}$ \\ ${ }^{1}$ Clinical Microbiology Department, Institute for Public Health Brod-Posavina County, Slavonski Brod \\ ${ }^{2}$ Neurosurgery section, Department of Surgery, GH, Slavonski Brod \\ ${ }^{3}$ Department of Anesthesiology and Intensive Care, GH, Slavonski Brod \\ ${ }^{4}$ Reference Center for Systemic Mycoses, Croatian National Institute for Public Health, Zagreb
}

*Corresponding author: Maja Tomić Paradžik, MD, Clinical Microbiology Department Institute for Public Health Brod-Posavina County, Vladimira Nazora 2A, Slavonski Brod 35-000, Croatia, Tel: +385-35-444-051; Fax: +385-35-440-244; E-mail: muski2aster@yahoo.com

Received date: June 12, 2015; Accepted date: July 15, 2015; Published date: July 22, 2015

Copyright: (c) 2015 Paradžik MT, et al. This is an open-access article distributed under the terms of the Creative Commons Attribution License, which permits unrestricted use, distribution, and reproduction in any medium, provided the original author and source are credited.

\section{Introduction}

T. asahii, a yeast from the Basidiomycetes class, is one of lifethreatening opportunistic pathogens, most prominently for granulocytopenic and immunocompromised, as well as AIDS, patients [1-5]. Cases of infection have also been documented in nongranulocytopenic and non-immunocompromised, surgery, as well long-term hospitalization, patients in ICUs [6-9]. The incidence of this pathogen as the causative agent of fungemia in neutropenic patients is more common in the Far East, most prominently in Japan where it is considered an agent of summertime hypersensitivity pneumonitis, condition reported solely in this area [6].

Genus Trichosporon belongs to the Trichosporonaceae family, with all species widely represented in nature and isolated from the soil, water, plants, as well as samples taken from mammals and birds. They tend to be present in the normal flora of the mouth, skin and nails, but also the causative agents of superficial and deep infection in humans $[8,10]$.

All pathogen species of the genus Trichosporon were once considered a single species $-T$. beigelii. Owing to their morphological, biochemical and molecular specificities on the levels of DNA/DNA and $26 \mathrm{~S}$ ribosomal RNA segments, however, T. beigelii was divided into separate species, 9 of which, at least, have the potential to cause a human infection: $T$. asahii (the most common causative agent of disseminated infections in man, rarely the agent of human or animal white piedra and a likely agent of summertime hypersensitivity pneumonitis in Japan), T. inkin (causative agent of white piedra in the genital region, rarely the agent of systemic infections such as endocarditis or peritonitis), T. asteroides (superficial lesions of the skin), $T$. cutaneum (skin lesions, white piedra in the armpit area), $T$. mucoides (rare systemic infections with a predilection to spread to the central nervous system, summertime hypersensitivity pneumonitis in Japan, onychomycosis), T. ovoides (white piedra of the scalp), $T$. loubieri (rare systemic infections isolated from the sputum of patients with cystic fibrosis), $T$. japonicum (pneumonitis, white piedra) and $T$. pullulans (skin lesions, white piedra) $[2,3,8,10]$.

Despite their being widespread in the environment, infections caused by Trichosporon asahii are extremely rare, mostly occurring in persons with an immune system or local immune reaction disorders $[4-6,8]$.

In most recorded cases of invasive trichosporonosis (63\%), the underlying morbidity is malignant diseases of the blood as the main predisposing factor [6].
Systemic infections caused by said pathogen are, consequently, most common in patients with acute leukemia, granulocytopenia, neutropenia, solid tumors, hemochromatosis, uremia and AIDS or undergoing corticosteroid therapy and chemotherapy $[4-6,10]$.

The main risk factors for contracting a $T$. asahii infection are the presence of external bodies such as IV catheters, urinary catheters or artificial valvulae $[7,8]$.

Despite the usual amphotericin B (AMB) therapy, systemic infections in malignant cases most commonly have a fatal result as this antifungal agent has shown limited in vitro efficacy in fighting the Trichosporon genus $[6,8,9,11]$.

Antifungals from the azole group in in vitro experiments have, on the contrary, shown good suppressant efficacy against this genus and their application has had satisfactory results in animal models. However, in in vitro activities they have shown questionable success $[9,11]$.

Results obtained by in vitro testing, as well as from animal models, call for a combined therapy against systemic trichosporonosis. It is recommended to combine amphotericin $B$ with caspofungin in severe cases of trichosporonosis $[6,11]$.

\section{Patient Report}

A young man of 22, with no significant illnesses in his heteroanamnesis, was admitted to an ICU unconscious, (GCS-3), after a severe poly- and neurotrauma with an injury to the frontal parietal area with the prolapse of the brain tissue.

After intubation and introduction of mechanical ventilation, severe bone trauma of the head was confirmed with a consequent malignant brain edema. Subarachnoid areas were filled with hemorrhagic content including gas bubbles, while subdural hematomas were present bilaterally frontally. After stabilization and radiology, the patient received neurology treatment and underwent osteoclastic craniotomy of the frontal region, revision of the lesion and plastic surgery of the frontal and frontal basal dura mater.

On two occasions, the patient was operated to have a Pudenz catheter built in due to developed hypertension hydrocephalus, and the cist in the frontal lobe evacuated.

Aside from the head trauma, the patient sustained a fracture of the left upper leg, which was treated by osteosynthesis, and chest contusion without rib fracture. 
Treatment course was complicated by numerous infections. The patient was treated with a series of broad-spectrum antibiotics (Cephalosporin, Carbapenem, Vancomycin). During his stay in the ICU, physical therapy was continually administered. After percutaneous tracheotomy and separation from the respirator, the patient regained consciousness, offered no verbal contact, had flaccid plegia of the right arm and spastic plegia of the left arm, flexion contracture of both legs, was fully immobile and lacked spontaneous motor coordination. Upon 3.5 months of ICU, the patient was moved to a specialized hospital for medical rehabilitation.

On a CAT scan check-up, done during rehabilitation, an encapsulate was noted on the right frontal side. The encapsulate imbibed the inserted contrast showing a visible lower liquid level with an upper air crescent sign consistent with an abscess accompanied by edema and subfacial herniation. The patient was transferred to a neurosurgery ward in General Hospital in Slavonski Brod. At the neurosurgery ward he was treated for brain abscess, shunt meningitis and hypertension hydrocephalus. Due to problems concerning the size of the urinal, the urinary catheter was left in place. During his stay, the patient experienced high febrility and increase in CRP 130, L 18, 6. Samples were taken for microbiological analysis (urine, hemoculture, cerebrospinal fluid). Due to developed shunt meningitis, internal drain was substituted by an external one.

In the liquor produced through the drainage system, there ensued a rise in the concentration of Proteus mirabilis and Streptococcus viridans. Antimicrobial therapy was, consequently, introduced according to an antibiogram. In the course of antimicrobial treatment, the patient became febrile again and his inflammation parameters grew. From the urine and blood hemoculture samples, a yeast-like fungus was isolated, which was subsequently confirmed to be Trichosporon asahii.

Microscopy findings of the hemoculture were sent to the neurosurgery ward, after which fluconazole was added to the therapy at a dose of $400 \mathrm{mg} /$ day with a recommendation of urinary catheter replacement/removal as a vital hygienic and therapeutic measure.

The introduction of the antifungal into the therapy and catheter replacement with a urinal brought about a drop in bodily temperature, $\mathrm{L}$ and CRP values and a clinically improved condition. After the completion of surgical treatment, the patient was again referred to a specialized facility for medical rehabilitation.

\section{Microbiology Diagnostics}

After a 24-hour incubation of the urine sample cultured by standard procedure-calibrated loop on blood agar, the yeast-like fungus multiplied. Direct microscopic preparation from the positive hemoculture (Bactec, Becton-Dickinson, UK) shows septate hyphae, arthroconidia and budding blastoconidia. After 24-hour incubation on blood culture media and Saboraud dextrose agar, white and creamy yellow colonies with powdery center and raised, deeply fissured edges multiplied (Figure 1). Individual colonies were transplanted to CHROMagar Candida (Biolife, Milan, Italy) and incubated at $35^{\circ} \mathrm{C} / 24$ hrs (Figure 2).

Microscopic preparation of the multiplied strain shows actual hyphae creating articular rectangular arthroconidia with rounded brim and dimensions 3-4 $\mathrm{mm} \times 4-7 \mathrm{~mm}$ (Figure 3).
As this was a case of an invasive non-albicans isolate, the strain was sent to the Reference Center for Systemic Mycoses, CNIPH, Zagreb, where $T$. asahii was, based on biochemical properties, confirmed.

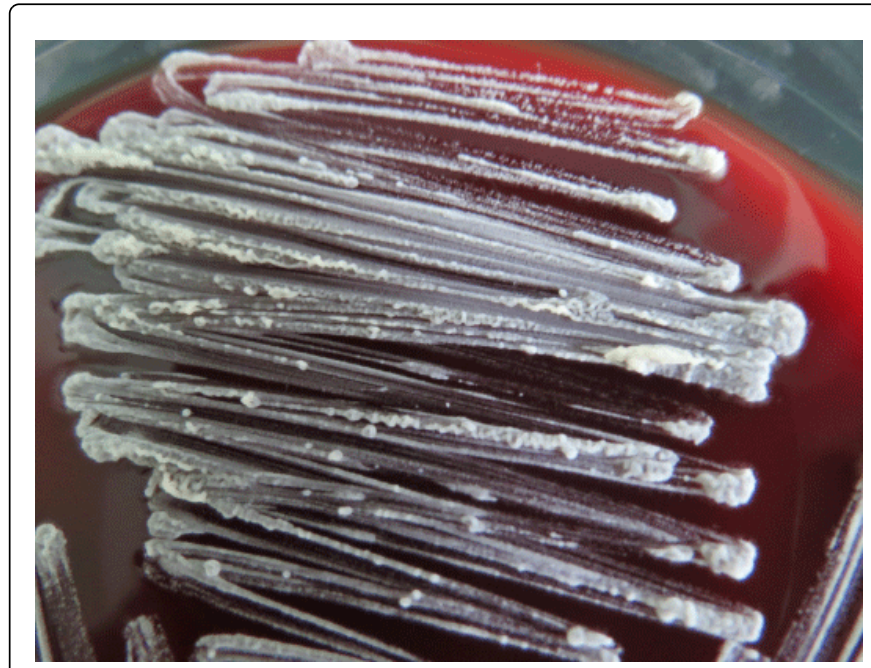

Figure 1: Trichosporon asahii on blood agar (Tryptic Soy Blood Agar Base sa 5\% sheep blood, Biolife, Italy) (Photographed at the Department of Clinical Microbiology, Institute of Public Health, Slavonski Brod, with a camera Canon PowerShot, SX 40HS).

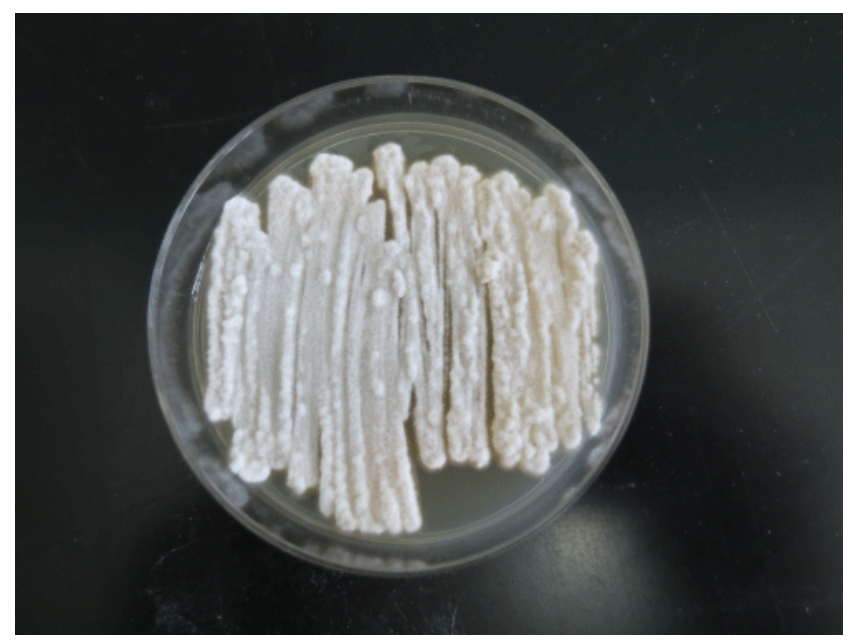

Figure 2: Trichosporon asahii on a selective medium for fungi and mold (Saboraud Dextrose agar, Biolife, Italy) (Photographed at the Department of Clinical Microbiology, Institute of Public Health, Slavonski Brod, with a camera Canon PowerShot, SX 40HS).

Isolate identification was based on classic biochemical methods, such as culturing on cyclohexamide agar and assimilation of glucose, sucrose, maltose, lactose, galactose and L-arabinose, but also ready-touse commercial identification systems, like ID $32 \mathrm{C}$ (BioMerieux).

Sensitivity to antifungal agents was detected using ATB FUNGUS 3 (BioMerieux). Results were read against CLSI recommendations, while the isolate demonstrated good sensitivity to 5 -fluorocitozin (MIK 4,0), fluconazole (MIK 1,0) and voriconazole (MIK 0,060), reduced 
sensitivity to itraconazole (MIK 0,250) and resistance to amphotericin B (MIK 4,0).

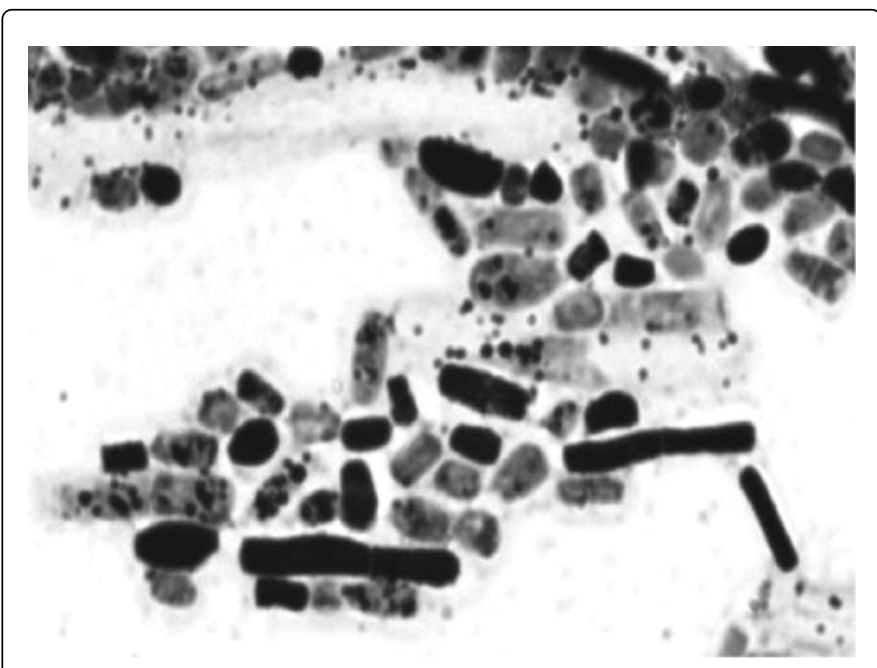

Figure 3: Microscopic perspective of Trichosporon asahii (Photographed at the Department of Pathology, GH (Dr. Josip Benčević), Slavonski Brod, microscope and camera Olympus, BX 41).

\section{Discussion and Conclusion}

Invasive infections caused by rarely pathogenic and opportunistic fungi have become a significant medical and therapeutic problem in immunocompromised and immunosuppressed patients. The first ever documented cases of invasive trichosporonosis date back to 1970s in patients with leukemia and have been rather sporadic to date $[2,8,10]$.

According to a retrospective study, invasive trichosporonosis develops in merely $0.9 \%$ patients suffering from acute leukemia, specifically more commonly in men [8]. In 1990s, two cases of invasive trichosporonosis were reported in India, again in immunocompromised individuals $[12,13]$.

One of the risk factors for contracting invasive trichosporonosis in immunocompromised persons today is found to be unregulated or insulin-dependent diabetes mellitus, as confirmed by Indian and Israeli cases $[1,6,12]$. The combination of multiple risk factors in immunocompromised patients such as presence of external bodies (catheters) and consequent circumvention of the local mucosal defense, long-term administration of broad-spectrum antibiotics and immune dysfunction in renal failure, favors the development of systemic infection by said pathogen, while the colonization of catheter and mucous membrane may lead to dissemination and formation of foci on remote organs [6,7].

The patient in this study, aged 22, was, prior to poly- and neurotrauma, healthy and immunocompetent, without any significant diseases in his medical history.

However, long-term hospitalization, presence of external bodies, artificial ventilation, previous complicating bacterial infections and consequent repeated administration of broad-spectrum antibiotics were the trigger for an infection caused by this opportunistic pathogen.

Documented pathway of fungemia development in this case was the urinary catheter and consequent bypassing of the local mucosal defense-an open door to the urinary system and penetration into the circulation accompanied by sepsis symptoms.

Owing to urinary catheter replacement with a urinal and extending the therapy to fluconazole, the results were exceptional clinical response and drop in all infection indicators. In in vivo experiments on animal models, azoles have proven to be much more effective in treating trichosporonosis than amphotericin B, though the combination of fluconazole and amphotericin B obtained better clinical response in documented cases of infection in immunocompetent persons $[6,8,10,14,15]$.

\section{References}

1. Rastogi VL, Nirwan PS (2007) Invasive trichosporonosis due to Trichosporon asahii in a non-immunocompromised host: a rare case report. Indian J Med Microbiol 25: 59-61.

2. Guého E, Improvisi L, de Hoog GS, Dupont B (1994) Trichosporon on humans: a practical account. Mycoses 37: 3-10.

3. Guého E, Smith MT, de Hoog GS, Billon-Grand G, Christen R, et al. (1992) Contributions to a revision of the genus Trichosporon. Antonie Van Leeuwenhoek 61: 289-316.

4. Girmenia C, Pagano L, Martino B, D,Antonio D, Fanci R, et.al. (2005) Invasive Infections Caused by Trichosporon Species and Geotrichum capitatum in Patients with Hematological Malignancies: a Retrospective Multicenter Study from Italy and Review of the Literature. J Clin Microbiol 43: 1818-1828.

5. Gross JW, Kan VL (2008) Trichosporon asahii infection in an advanced AIDS patient and literature review. AIDS 22: 793-795.

6. Wolf DG, Falk R, Hacham M, Theelen B, Boekhout T, et al. (2001) Multidrug-resistant Trichosporon asahii infection of nongranulocytopenic patients in three intensive care units. J Clin Microbiol 39: 4420-4425.

7. Sood S, Pathak D, Sharma R, Rishi S (2006) Urinary tract infection by Trichosporon asahii. Indian J Med Microbiol 24: 294-296.

8. Warkentien TE, Maves RC, Hale BR (2009) Trichosporon Infections. Medscape.

9. Wolf DG, Fark R, Hachman M, Theclen B, Boekhout T, et.al. (2001) Multidrug resistant Trichosporon asahii infection of nongranulocytopenic patients in intensive care unit. J Clin Microbiol 39: 4420-4425.

10. Sutton DA, Fothergill AW, Rinaldi MG (998) Guide to Clinically Significant Fungi. Williams \& Wilkins, Baltimore.

11. Li H, Lu Q, Wan Z, Zhang J (2010) In vitro combined activity of amphotericin $\mathrm{B}$, caspofungin and voriconazole against clinical isolates of Trichosporon asahii. Int J Antimicrob Agents 35: 550-552.

12. Kumar S, Bandyopadhyay M, Mondal S, Pal N (2011) A Rare Case of Nosocomial Urinary Tract Infection due to Trichosporon asahii. J Glob Infect Dis 3: 309-310.

13. Chakrabarti A, Marhawa RK, Mondal R, Trehan A, Gupta S, et al. (2002) Generalized lymphadenopathy caused by Trichosporon asahii in a patient with Job's syndrome. Med Mycol 40: 83-86.

14. Takamura S, Oono T, Kanzaki H, Arata J (1999) Disseminated Trichosporonosis with Trichosporon asahii. Eur J Dermatol 9: 577-579.

15. Richardson DM (2008) Fungal Infection and Critical Care Medicine. Gilead Sciences Ltd., Brandi, London. 